\title{
New data on encyrtid (Hymenoptera: Chalcidoidea: Encyrtidae) parasitoids of Coccoids (Hemiptera: Coccoidea) from Turkey, with description of five new species
}

\author{
George Japoshvili
}

\begin{abstract}
Japoshvili, G. 2012: New data on encyrtid (Hymenoptera: Chalcidoidea: Encyrtidae) parasitoids of Coccoids (Hemiptera: Coccoidea) from Turkey, with description of five new species. - Entomol. Fennica 23: 72-82.

Parasitoids of coccoids were surveyed using malaise traps at the Golcuk Natural Park (Isparta Province), Turkey, in 2009 and 2010. More than 40 species of parasitoids were collected, with twelve being new records for Turkey, of which five, Anagyrus descriptus sp. n., A. alienus sp. n., Aschitus golcukus sp. n., Charitopus bulentyasari sp. n., and Ch. ismailkaracai sp. n. (Hymenoptera: Encyrtidae), are described as new species.

G. Japoshvili, Entomology and Biocontrol Research Centre, Agricultural University of Georgia, $13 \mathrm{~km}$ David Aghmashenebeli Alley, Tbilisi, 0131, Georgia; E-mail: giorgij70@yahoo.com
\end{abstract}

Received 5 December 2010, accepted 11 August 2011

\section{Introduction}

Encyrtidae is the most speciose group of parasitoids attacking scales and psyllids. Members of this family are important in biological control. More than 400 encyrtid species have been used or are used today for suppression of various crop pests (Japoshvili \& Noyes 2006). There are more than 1270 described species of encyrtids in the Palaearctic Region (Trjapitzin 1989, Yasnosh \& Japoshvili 1999, Japoshvili 2005, 2007, Japoshvili \& Karaca 2004, Japoshvili \& Noyes 2005a, 2006, Japoshvili \& Abrantes 2006). The first comprehensive account of encyrtid parasitoids of Coccoids from Turkey was given in Öncüer's (1991) catalogue, where he recorded 27 species of encyrtids and 17 species of aphelinids. The number of encyrtid species was later increased to 50 by Trjapitzin \& Doganlar (1997). Recent studies in Turkey have found an additional 82 encyrtid species (Japoshvili \& Karaca 2002, 2004, Karaca \& Japoshvili 2002, Japoshvili et al.
2004, Uygun et al. 2004, Japoshvili 2005, Japoshvili \& Noyes 2005a,b, 2006, Kaydan et al. 2006, Japoshvili \& Celik 2010, Kaydan \& Japoshvili 2010).

The purpose of this study was to identify encyrtid parasitoids of Coccoids using malaise traps. The genera that are discussed in this study are recorded as parasitoids of Coccoids, without exception.

\section{Material and methods}

Insect samples were collected from March to October in 2009 and 2010, using malaise traps (Townes 1972), which were supplied by BandS Entomology-The Net Suppliers (UK). The traps were placed in two locations: Pilav Tepe (N: $37^{\circ}$ 43'19.35”, E: $30^{\circ} 29^{\prime} 19.74 ”, 1,520 \mathrm{~m}$ a.s.1.) and Acacia reforested area, near main entrance at Golcuk Natural Park (N: $37^{\circ} 44^{\prime} 12.33$ ', E: $30^{\circ}$ 29'24.00", 1,414 m a.s.1.). The traps were 


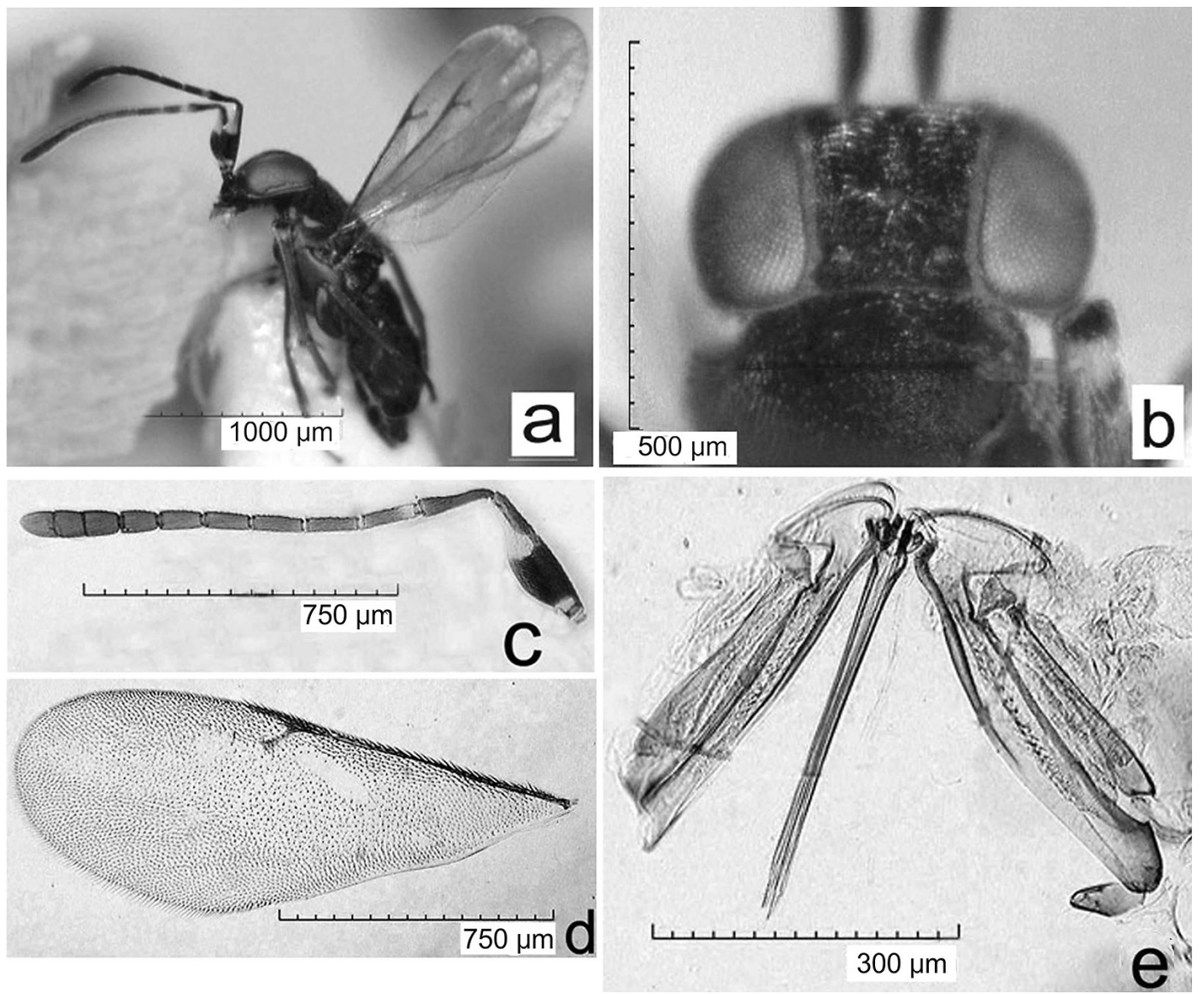

Fig. 1. Anagyrus alienus sp. n. female. - a. Body. - b. Head. - c. Antenna. - d. Fore wing. - e. Ovipositor.

checked and material was collected every 10 days. Additionally, from March to July, I set malaise trap in a rocky area (N: $37^{\circ} 42^{\prime} 52.51^{\prime}, \mathrm{E}: 30^{\circ}$ 29'47.42", 1,430 m a.s.1.), also checked every 10 days. Captured insects were sorted into orders, and then hymenopterans were sorted into superfamilies and families. Specimens in the superfamily Chalcidoidea were critical point dried to preserve morphological features. Encyrtid specimens were card mounted and slide mounted according to Noyes (2010). Identification was done by the author using various identification keys and literature sources (Trjapitzin 1989; Noyes \& Hayat 1994; Guerrieri \& Noyes 2000; Hayat 2006). Descriptions of new species of parasitoids were prepared according to Noyes \& Hayat (1994).

During these studies, available material from various museums were also examined, including the Smithsonian Institution, the Madrid Natural History Museum, the Natural History Museum in London and the St. Petersburg Entomology Collection of the Institute of Zoology.

The following abbreviations are used in the text: AOL, distance between posterior and anterior ocelli; EL, maximum eye length; EOL, ovipositor length (exserted part); FV, minimum frontovertex width; $F_{1} L, F_{2} L$, etc., first funicle segment length, second funicle segment length, etc.; $F_{1} W, F_{2} W$, etc., first funicule segment width, second funicule segment width, etc.; FWL, length of forewing; FWW, width of forewing; GL, maximum length of gonostylus (= third valvula); GOL, abdomen length including exserted part of ovipositor; HW, maximum head width; $M$, length of marginal vein; $M F$, the length of the longest marginal setae on the forewing; MS, malar space (shortest distance from eye to 
Table 1. Differences between Anagyrus alienus Japoshvili sp. $\mathbf{n}$. and A. zaitzevi Trjapitzin. Abbreviations: FV, minimum frontovertex width; $\mathrm{AOL}$, distance between posterior and anterior ocelli; POL, posterior ocellar line (= the shortest distance between posterior ocelli).

\begin{tabular}{|c|c|}
\hline A. alienus sp. $\mathbf{n}$. & A. zaitzevi \\
\hline FV $0.41 \times$ as broad as head & FV $0.33 \times$ as broad as head width \\
\hline $\begin{array}{l}\text { Ocelli forming slightly obtuse angle } \\
\mathrm{AOL}<\mathrm{POL}\end{array}$ & $\begin{array}{l}\text { Ocelli forming slightly acute angle } \\
\mathrm{AOL}=\mathrm{POL}\end{array}$ \\
\hline $\begin{array}{l}\text { Midtibial basitarsus almost as long as midtibial spur } \\
\text { or slightly longer }\end{array}$ & $\begin{array}{l}\text { Midtibial basitarsus } 1.2-1.3 \times \text { as long } \\
\text { as midtibial spur }\end{array}$ \\
\hline Only basal half of flagellum $F_{1}$ whitish & Flagellum white or yellowish \\
\hline Antennae with scape about $3.3 \times$ as long as broad & $\begin{array}{l}\text { Antennae with scape about } 2.7 \times \text { as long as broad } \\
\text { Clava } 3 \times \text { as long as broad }\end{array}$ \\
\hline Clava $3.8 \times$ as long as broad & Clava $3 \times$ as long as broad \\
\hline
\end{tabular}

mouth margin); MT, length of midtibia; MTS, length of midtibial spur; MTT, length of midbasitarsus; OCL, occipital ocellar line (distance of posterior ocellus from occipital margin); OD, greatest diameter of an ocellus; OL, ovipositor length; OOL, ocular-ocellar line (shortest distance between posterior ocellus and adjacent eye margin); P, length of postmarginal vein; PL, pedicel length; POL, posterior ocellar line (= the shortest distance between the posterior ocelli); PW pedicel width; S, length of stigmal vein; SL, scape length; SW, scape width; ThL, thorax and head length together.

Illustrations were made by using a Hirox KH7700 digital microscope. The holotype and paratypes and other voucher specimens are deposited in the collection at the Entomology and Biocontrol Research Centre, Agricultural University of Georgia, Tbilisi, Georgia. New records are marked with a double asterisk.

\section{Descriptions of new species of the family Encyrtidae}

\subsection{Anagyrus alienus sp. n. Figs. 1a-e}

Material examined. Type material: Holotype + , Turkey, Isparta, Golcuk Natural Park, Pilav Tepe, $\mathrm{N}: 37^{\circ} 43^{\prime} 19.35^{\prime}$ ', E: $30^{\circ} 29^{\prime} 19.74 ”, 1,520 \mathrm{~m}$, 21.V.2009, leg. G. Japoshvili and H. Celik (slide).

Female. Length of holotype $1.7 \mathrm{~mm}$ (Fig. 1a).

All body dark brown, almost black; only sides of mesoscutum and scutellum with very small yellow spots on sides; mesopleuron orange; all body covered with densely silver bristles; eyes with orange band around; coxae with brown ventral surface and whitish on dorsal; legs yellowish; fore, middle legs and hind femora brown with one whitish line across; only hind tibia completely brownish.

Head 2.1× as broad as frontovertex (Fig. 1b); ocelli forming slightly obtuse angle; antennae with scape about $3.3 \times$ as long as broad, pedicel about $4.3 \times$ as long as broad (Fig. 1c).

Relative measurements (holotype). $\mathrm{HH}$ 497.5; HW 570.2; FV 237; MS 128.8; EL 384.6; AOL 80; POL 105.5; OOL 34; OD 30; OCL 28.8; THL 711; GOL 884; SL 335; SW 100.4; PL 167.3; PW 39.3; F L 141.5; F W 31.6; F L 129.9; $\mathrm{F}_{2} \mathrm{~W} 32.4 ; \mathrm{F}_{3} \mathrm{~L} 130.8 ; \mathrm{F}_{3} \mathrm{~W} 34.3 ; \mathrm{F}_{4} \mathrm{~L} 105.9 ; \mathrm{F}_{4} \mathrm{~W}$ 39; $\mathrm{F}_{5} \mathrm{~L} 104.9 ; \mathrm{F}_{5} \mathrm{~W} 43.8 ; \mathrm{F}_{6} \mathrm{~L} 86.2 ; \mathrm{F}_{6} \mathrm{~W} 52.7 ; \mathrm{CL}$ 235.6; CW 62; FWW 575.7; FWL 1517.7; M 118.35; P 131.7; S 98 (Fig. 1d); OL 453.6; GL 70.3 (Fig. 1e); MT 693.6; MTS 186; MTT 192.9.

Comment. The closest species to Anagyrus alienus Japoshvili sp. n. is A. zaitzevi Trjapitzin. Females of these species can be separated by the characters given in Table 1.

Etymology. The species is named according Latin name "alienus", which means "different".

\subsection{Anagyrus descriptus sp. n. Figs. 2a-e}

Material examined. Type material: Holotype $q$, Turkey, Isparta, Golcuk Natural Park, Acacia reforested area, N: $37^{\circ} 44^{\prime} 12.33^{\prime \prime}, \mathrm{E}: 30^{\circ}$ 29'24.00”, 1,414 m, 14.VIII.2009, leg. G. Japoshvili and H. Celik (slide).

Female. Length of holotype $1.2 \mathrm{~mm}$ (Fig. 2a).

Head with FV dark orange color; frons with brown spot on each side of toruli; genae and 

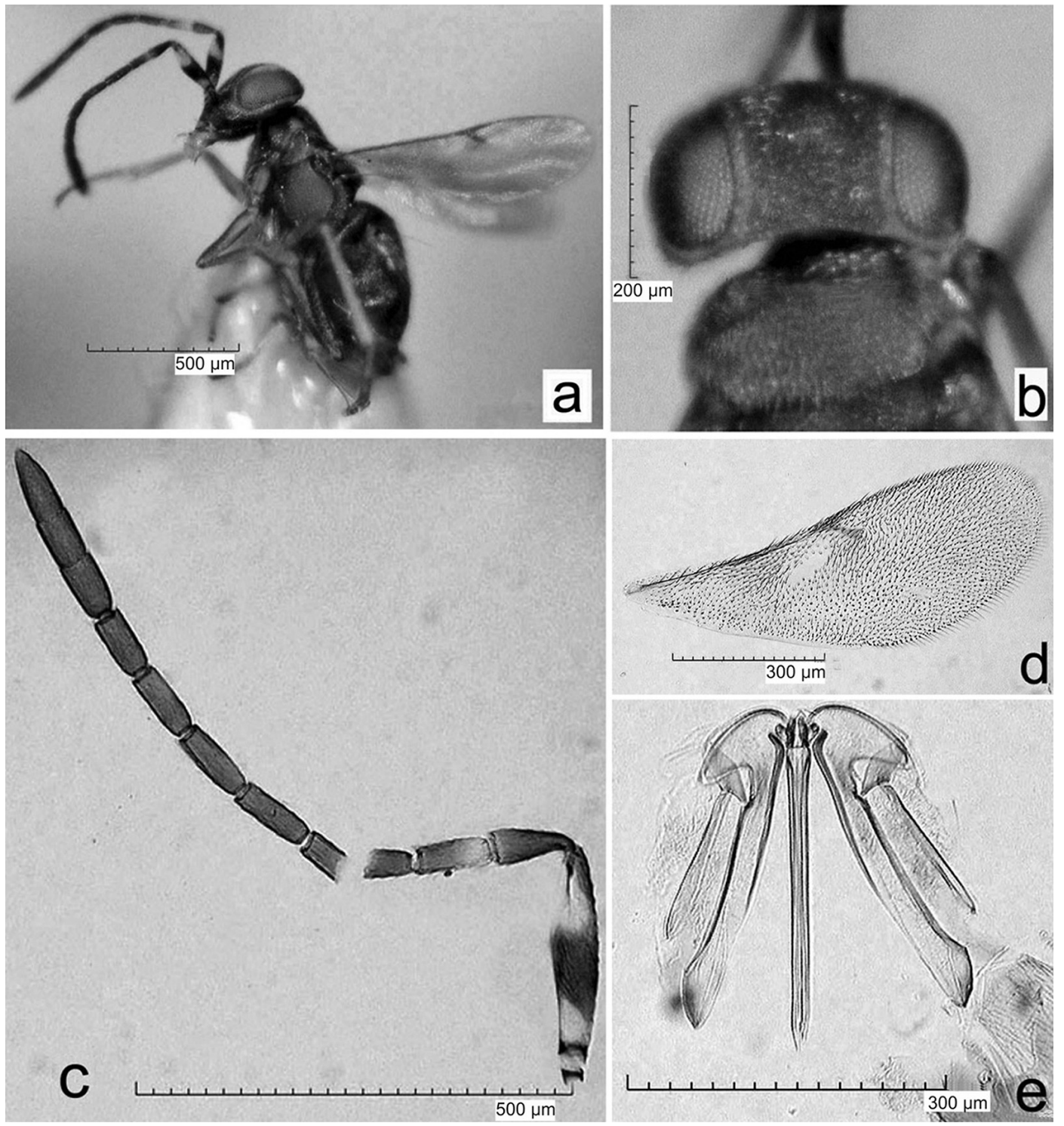

Fig. 2. Anagyrus descriptus sp. n. female. - a. Body. - b. Head. - c. Antenna. - d. Fore wing. - e. Ovipositor.

malar space brown except yellow band around eyes; all body dark orange color, only tegulla with basal half whitish and apically with brown spot; coxae yellow but infuscated; legs yellow but front and back sides with brown lines across; midtibia yellow; scape basal $1 / 5$ and apical $2 / 5$ whitish, middle $2 / 5$ brown; $F_{1}$ basal half whitish and all other segments brown.

Head $1.8 \times$ as broad as frontovertex (Fig. 2b); ocelli forming slightly obtuse angle; antennae with scape about $4 \times$ as long as broad, pedicel about $2.4 \times$ as long as broad (Fig. 2c).
Relative measurements (holotype). HH 370; HW 403.6; FV 207; MS 112; EL 256; AOL 61; POL 81; OOL 42; OD 22; OCL 27.7; THL 428,6; GOL 620; EOL 52.

Additional relative measurements. SL 229.8; SW 55.3; PL 91.5; PW 38.1; F L 89; $F_{1} W$ 31; F $\mathrm{F}_{2}$ $88 ; \mathrm{F}_{2} \mathrm{~W} 29.6 ; \mathrm{F}_{3} \mathrm{~L} 86.5 ; \mathrm{F}_{3} \mathrm{~W} 31 ; \mathrm{F}_{4} \mathrm{~L} 89 ; \mathrm{F}_{4} \mathrm{~W} 31$; $\mathrm{F}_{5} \mathrm{~L}$ 82.2; $\mathrm{F}_{5} \mathrm{~W} 30.5 ; \mathrm{F}_{6} \mathrm{~L} 76 ; \mathrm{F}_{6} \mathrm{~W} 31.6$; CL 206.5; CW 38.5; FWW 301.9; FWL 857.2; M 50.9; P 60.6; S 65.2 (Fig. 2d); OL 338.7; GL 59.5 (Fig. 2e); MT 459; MTS 159; MTT 149.8.

Comment. The closest species to Anagyrus 
Table 2. Differences between Anagyrus descriptus Japoshvili sp. n. and A. matritensis (Mercet). Abbreviations: $F_{1}, F_{2}$, etc., first funicle segment, second funicle segment, etc.; $F V$, minimum frontovertex width; AOL, distance between posterior and anterior ocelli; POL, posterior ocellar line (= the shortest distance between posterior ocelli).
A. descriptus sp. $\mathbf{n}$.
A. matritensis
$F_{1}$ basal half whitish and all other segments brown
FV $0.5 \times$ as broad as head
Ocelli forming slightly obtuse angle
$\mathrm{AOL}<\mathrm{POL}$
Eyes $2.3 \times$ as long as malar space
Scape $4 \times$ as long as broad
Pedicel $2.4 \times$ as long as broad; $F_{6} 2.4 \times$ as long
as broad
Head wider than high
Legs yellow but front and back sides
with brown lines across
Marginal vein shorter than postmarginal
$F_{2}$ and $F_{3}$ with basal half whitish
$F \bigvee 0.4 \times$ as broad as head
Ocelli forming slightly acute angle
AOL $=P O L$
Eyes more than $2.5 \times$ as long as malar space
Scape $3.3 \times$ as long as broad
Pedicel $4.5 \times$ as long as broad; $F_{6} 2 \times$ as long
as broad
Head as broad as high
Legs with dark parts but they don't form
lines across
Marginal vein almost as long as postmarginal

descriptus Japoshvili sp. n. is A. matritensis (Mercet). Females of these species can be separated by the characters given in Table 2 .

Etymology. The species is named according Latin name "descriptus", which means "well organized" or "described".

\subsection{Aschitus golcukus sp. n. Figs. 3a-e}

Material examined. Type material: Holotype + , Turkey, Isparta, Golcuk Natural Park, Acacia reforested area, $\mathrm{N}: 37^{\circ} 44^{\prime} 12.33^{\prime \prime}, \mathrm{E}: 30^{\circ}$ 29'24.00', 1,414 m, 28.VI.2009, leg. G. Japoshvili and H. Celik (slide).

Female. Length of holotype without exserted part of ovipositor $1.6 \mathrm{~mm}$ (Fig. 3a).

Head yellow, only behind posterior ocelli and eyes with brown band; pronotum, mesoscutum, scutellum and axillae brownish yellow with brown stripe (line) in the middle of pronotum, mesoscutum and scutellum; mesopleuron almost brown; gaster brown with tergites back side with yellow line; above the cerci in the middle $1 / 3$ almost yellow; all legs brownish yellow; scape brown with ventral light line and pedicel light brown; all flagellum and clava dark brown.

Head 2.8× as broad as frontovertex (Fig. 3b); ocelli forming slightly obtuse angle; antennae with scape about $2.2 \times$ as long as broad, pedicel about $1.64 \times$ as long as broad (Fig. 3c).

Relative measurements (holotype): HW 517,7; FV 183; MS 161.1; EL 265.1; AOL 50; POL 81; OOL 27; OD 23.8; OCL 29.6; THL 533.5; GOL 797.2; EOL 63.3.

Additional relative measurements: $\mathrm{HH} 404$; SL 247.9; SW 111.6; PL 68.8; PW 42; F L 42.9; $\mathrm{F}_{1} \mathrm{~W} 41 ; \mathrm{F}_{2} \mathrm{~L} 38 ; \mathrm{F}_{2} \mathrm{~W} 47.9 ; \mathrm{F}_{3} \mathrm{~L} 42.9 ; \mathrm{F}_{3} \mathrm{~W} 56.1$; $\mathrm{F}_{4} \mathrm{~L}$ 43.7; $\mathrm{F}_{4} \mathrm{~W} 61.8 ; \mathrm{F}_{5} \mathrm{~L} 44.9 ; \mathrm{F}_{5} \mathrm{~W} 66 ; \mathrm{F}_{6} \mathrm{~L}$ 45.6;

Table 3. Differences between Aschitus golcukus Japoshvili sp. n. and A. neoacanthococci Myartseva. Abbreviations: AOL, distance between posterior and anterior ocelli; $\mathrm{OD}$, greatest diameter of ocellus; $\mathrm{F}_{5}$, fifth funicle segment.

A. golcukus sp. $\mathbf{n}$.

Ocelli forming slightly obtuse angle

$\mathrm{AOL}>\mathrm{OD}$

Scape $2.2 \times$ as long as broad

Pedicel about $1.64 \times$ as long as broad

Marginal slightly more than $2 \times$ as long as postmarginal

$\mathrm{F}_{5}$ dark brown

\section{A. neoacanthococci}

Ocelli forming slightly acute angle

$\mathrm{AOL}<\mathrm{OD}$

Scape slightly less than $2 \times$ as long as broad

Pedicel slightly less than $2 \times$ as long as broad

Marginal vein slightly less than $1.5 \times$ as long

as postmarginal

$F_{5}$ yellow 

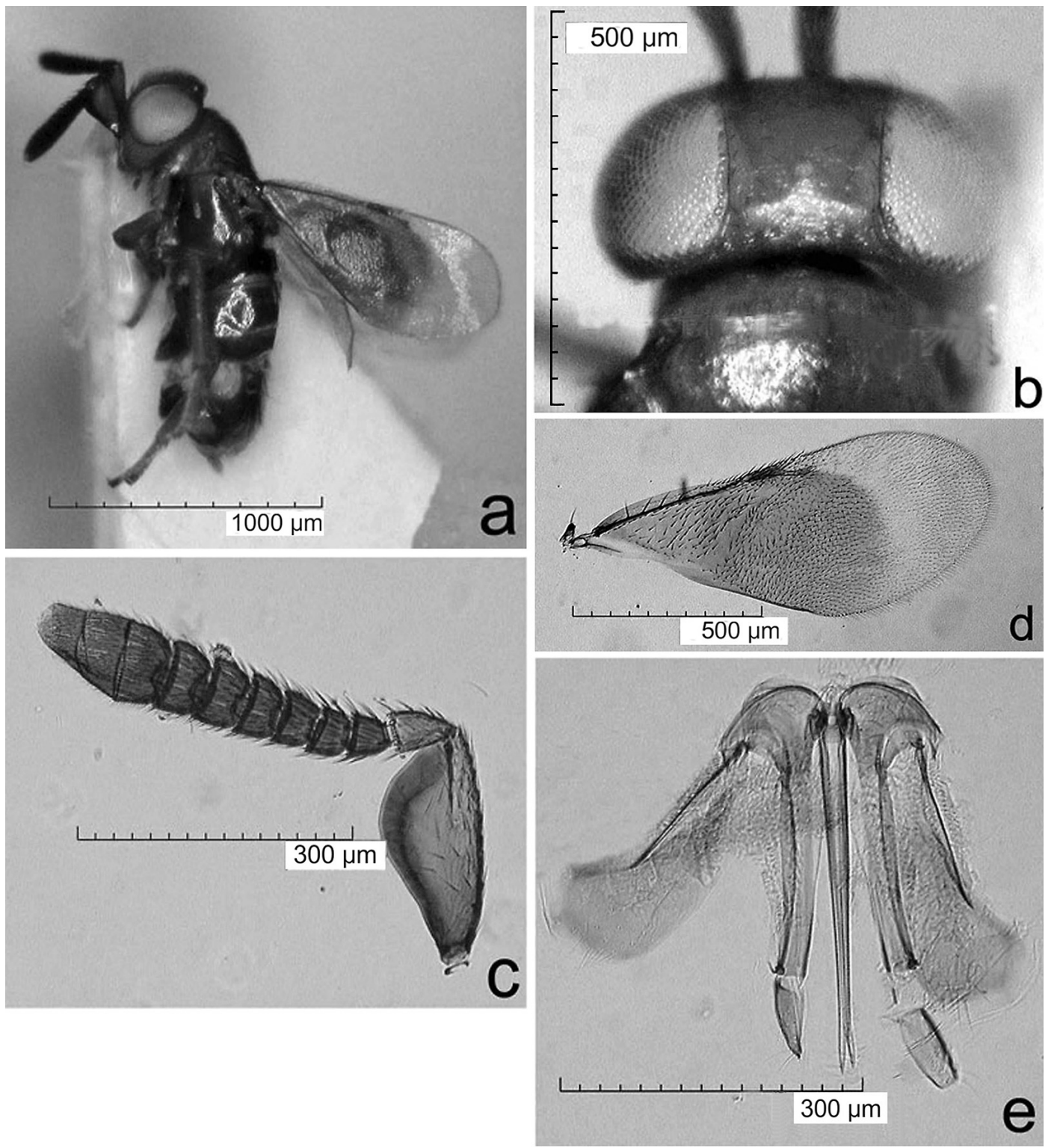

Fig. 3. Aschitus golcukus sp. n. female. - a. Body. - b. Head. - c. Antenna. - d. Fore wing. - e. Ovipositor.

F ${ }_{6}$ 74.6; CL 156.3; CW 85.9; FWW 446.8; FWL 1063; M 110.8; P 51; S 95.7 (Fig. 3d); OL 395.8; GL 82 (Fig. 3e); MT 440.6; MTS 125.5; MTT 125.3.

Comment. The closest species to Aschitus golcukus Japoshvili sp. n. is A. neoacanthococci Myartseva. Females of these species can be separated by the characters given in Table 3 .

Etymology. The species is named according lake name "Golcuk", around which the species was found.

\subsection{Charitopus bulentyashari sp. n. Figs. 4a-c}

Material examined. Type material: Holotype ${ }_{+}$, Turkey, Isparta, Golcuk Natural Park, Acacia reforested area, $\mathrm{N}: 37^{\circ} 44^{\prime} 12.33^{\prime \prime}, \mathrm{E}: 30^{\circ}$ 29'24.00”, 1,414 m, 03.VII.2009, leg. G. Japoshvili and H. Celik (card mounted); Paratype, + , Acacia reforested area, 12.VII.2009, leg. G. Japoshvili and H. Celik (slide).

Female. Length of holotype $1.41 \mathrm{~mm}$.

Head dark brown with metallic violet-silver 
Table 4. Differences between Charitopus bulentyasari Japoshvili sp. n., Ch. fulviventris Förster and Ch. eristoi Japoshvili. Abbreviations: FV, minimum frontovertex width; $F_{1}$, first funicle segment.

\begin{tabular}{|c|c|c|}
\hline Ch. bulentyasari sp. $\mathbf{n}$. & Ch. fulviventris & Ch. eristoi \\
\hline All coxae and femora brown & Fore and hind femora brown & All coax and femora yellow \\
\hline Head $2 \times$ as broad as FV & Head $2.4 \times$ as broad as FV & Head $2.2 \times$ as broad as FV \\
\hline Pedicel $1.4 \times$ as long as $F_{1}$ and & Pedicel 1.6 as long as $F_{1}$ and & Pedicel 1.3 as long as $F_{1}$ \\
\hline $2.7 \times$ as long as broad & $3 \times$ as long as broad & and $2.4 \times$ as long as broad \\
\hline Scape $3.6 \times$ as long as pedicel & Scape $2.5 \times$ as long as pedicel & Scape $3.4 \times$ as long as pedicel \\
\hline Exserted part of ovipositor & Exserted part of ovipositor & Exserted part of ovipositor \\
\hline $0.1 \times$ as long as gaster & $0.2 \times$ as long as gaster & $0.05 \times$ as long as gaster \\
\hline
\end{tabular}

reflection; pronotum, mesopleuron, mesoscutum, scutellum, axillae, sides of abdomen, metanotum, propodeum, all coxae and femora brown with metallic violet-golden reflection. Fore tibia with basal $1 / 4$ brown; midtibia with basal half brown and hind tibia with slight infuscation at the basal $1 / 4$ to $1 / 2$ dark brown; antennae all dark brown with
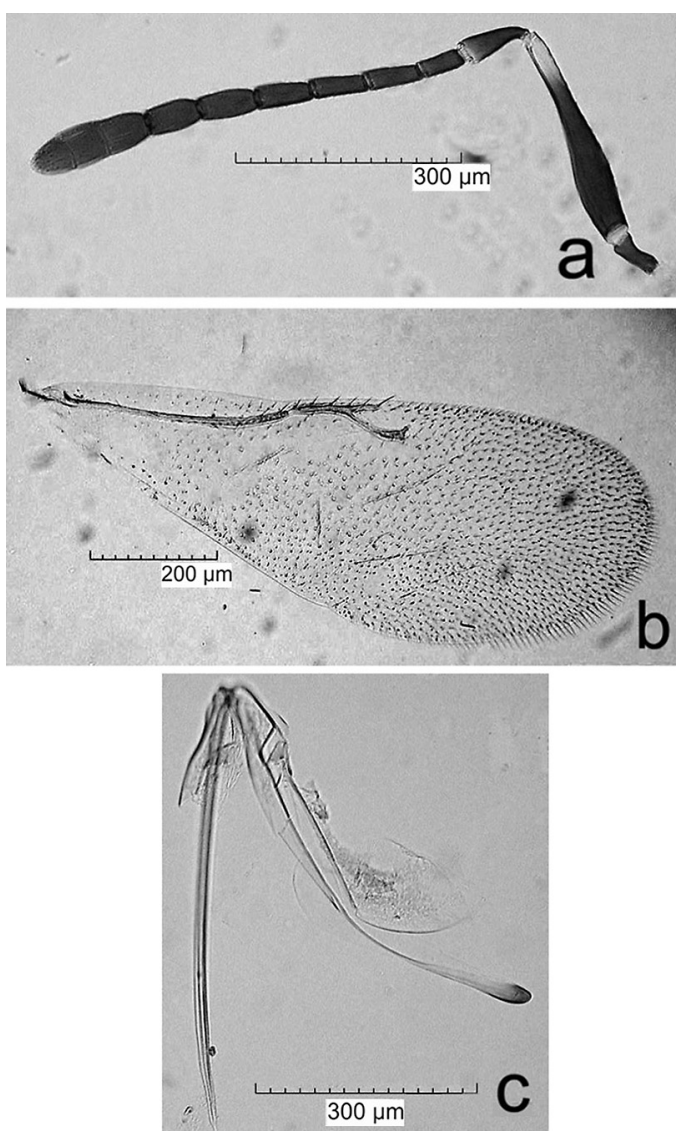

Fig. 4. Charitopus bulentyasari sp. n. female. - a. Antenna. - b. Fore wing. - c. Ovipositor. metallic violet-golden reflection, only apical $1 / 5$ of scape light. Occipital margin very sharp.

Head $2 \times$ as broad as frontovertex; ocelli forming obtuse angle; antennae with scape about $6 \times$ as long as broad, pedicel about $2.7 \times$ as long as broad (Fig. 4a).

Relative measurements (holotype): $\mathrm{HH} 353$; HW410.6; FV 173.5; MS 118.8; EL 224.4; AOL 34.5; POL 67.9; OOL 27; OD 21; OCL 23; THL 418; GOL 801.8; EOL 77.

Additional relative measurements (paratype): HH 372; HW 457.6; FV 200; MS 181; EL 238; SL 313; SW 53; PL 92; PW 34; F L 64; F W23.5; $\mathrm{F}_{2} \mathrm{~L} 74.7 ; \mathrm{F}_{2} \mathrm{~W} 24.7 ; \mathrm{F}_{3} \mathrm{~L}$ 75; $\mathrm{F}_{3} \mathrm{~W} 26 ; \mathrm{F}_{4} \mathrm{~L}$ 82.2; $\mathrm{F}_{4} \mathrm{~W} 30.7 ; \mathrm{F}_{5} 179 ; \mathrm{F}_{5} \mathrm{~W} 35.7 ; \mathrm{F}_{6} \mathrm{~L} 74.8 ; \mathrm{F}_{6} \mathrm{~W}_{40.7}$; CL 169; CW 54; FWW 384; FWL 1044; M 97; P 45.8; S 98.8 (Fig. 4b); OL 637; GL 286 (Fig. 4c); MT 377; MTS 55.7; MTT 91.

Comment. The closest species to Charitopus bulentyasari Japoshvili sp. n. are Ch. fulviventris Förster, 1860 and Ch. eristoi Japoshvili. Females of these species can be separated by the characters given in Table 4.

Etymology. The species is named after Prof. Bulent Yasar who is a well known Coccoidologist from Turkey.

\subsection{Charitopus ismailkaracai sp. n. Figs. 5a-c}

Material examined. Type material: Holotype ${ }_{+}$, Turkey, Isparta, Golcuk Natural Park, Acacia reforested area, $\mathrm{N}: 37^{\circ} 44^{\prime} 12.33^{\prime \prime}, \mathrm{E}: 30^{\circ}$ 29'24.00", 1,414 m, 03.VII.2009, leg. G. Japoshvili and H. Celik (card mounted); Paratypes, Acacia reforested area, 25.VI.2009, 2 q, leg. H. Celik (slide); Acacia reforested area, 03.VII.2009, 19, H. Celik; Acacia reforested area, 14.VIII.2009, 2 , , leg. G. Japoshvili and H. Celik. 

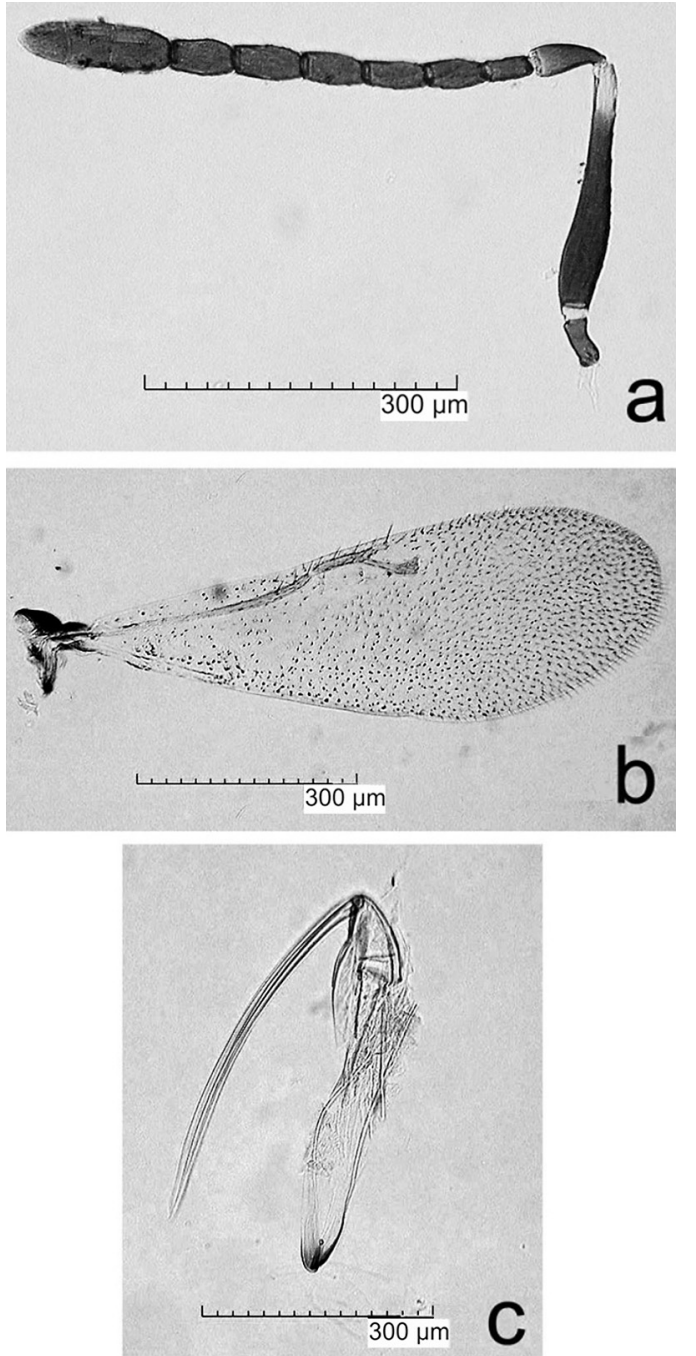

Fig. 5. Charitopus ismailkaracai sp. $\mathbf{n}$. female. $-\mathbf{a}$. Antenna. - b. Fore wing. - c. Ovipositor.

Female. Length of holotype $1.4 \mathrm{~mm}$.

Head dark brown with metallic violet-golden reflection; pronotum and mesoscutum with violet-golden-green, axillae and scutellum with violet-silver, mesopleuron, metanotum, propodeum and sides of abdomen, with silver reflection; fore and mid coxae brown, hind coax infuscated to brownish; fore and mid femora brown; hind femora completely yellow, in some specimens can be with ring like infuscation at basal part; fore and hind tibia yellow, midtibia with ring like infuscation at the basal part; antennae all brown, only scape with apical $1 / 6$ to $1 / 7$ light. Occipital margin very sharp.
Head $2.4 \times$ as broad as frontovertex; ocelli forming obtuse angle; antennae with scape about $5.7 \times$ as long as broad, pedicel about $2.9 \times$ as long as broad (Fig. 5a).

Relative measurements (holotype): $\mathrm{HH}$ 397.5; HW 426; FV 179; MS 140.8; EL233.7; AOL 40; POL 78.5; OOL 33; OD 21.4; OCL 12; THL 416; GOL 767; EOL 65.

Additional relative measurements (paratype): HH 310.8; HW 349.5; FV 179; MS 132.6; EL 190.5; SL 245.5; SW 43; PL 73.5; PW 25.2; F $L_{1}$ 48; $\mathrm{F}_{1} \mathrm{~W} 19 ; \mathrm{F}_{2} \mathrm{~L}$ 56.5; $\mathrm{F}_{2} \mathrm{~W} 27.6 ; \mathrm{F}_{3} \mathrm{~L} 60 ; \mathrm{F}_{3} \mathrm{~W}$ 25.7; $\mathrm{F}_{4} \mathrm{~L} 64.8 ; \mathrm{F}_{4} \mathrm{~W} 28.8 ; \mathrm{F}_{5} \mathrm{~L}$ 65.8; $\mathrm{F}_{5} \mathrm{~W} 33.4$; $\mathrm{F}_{6} \mathrm{~L}$ 64.8; $\mathrm{F}_{6} \mathrm{~W}$ 35.3; CL 149.8; CW 49; FWW 278.6; FWL 824; M 83; P 54; S 76.2 (Fig. 5b); OL 519; GL 142.6 (Fig. 5c); MT 323.5; MTS 38.7; MTT 51.3.

Comment. The closest species to Charitopus ismailkaracai Japoshvili sp. n. is Ch. desertus Myartseva. Females of these species can be separated by the characters given in Table 5 .

Etymology. The species is named after Prof. Ismail Karaca who made valuable contributions to biocontrol in Turkey.

\section{List of other species recorded in Golcuk Natural Park}

In this study more than 40 encyrtid parasitoid species of coccoids were found. Seven species were newly recorded for the Turkish fauna (indicated by $* *$ in the list below), in addition to the five species described as new to science above. Specimens identified to species are listed below. Baeocharis sp., Coccoidencyrtus sp., several Ericydnus spp., Subprionomitus sp., Trichomasthus $\mathrm{sp}$. are not included in the list. For the two main study localities at the Golcuk Natural Park, i.e. Acacia reforested area and Pilav Tepe, the abbreviations of $\mathrm{AR}$ and $\mathrm{PT}$, respectively, are used below. Abbreviations of GJ and $\mathrm{HC}$ are used for $\mathrm{G}$. Japoshvili and H. Celik, respectively.

Anagyrus aligarhensis Agarwal and Alam, 1959

Material examined: AR, 14.VIII.2009, 1ठ, leg. GJ \& HC; AR, 24.VIII.2009, 2 స, leg. GJ \& HC; AR, 03.IX.2009, 20̂, leg. GJ \& HC; AR, 14.IX.2009, 1ㅇ, 1§, leg. GJ \& HC.

**Anagyrus securicornis Domenichini, 1953

Material examined: PT, 24.VIII.2009, 19, 
Table 5. Differences between Charitopus ismailkaracai Japoshvili sp. n. and Ch. desertus Myartseva. Abbreviation: $\mathrm{F}_{6}$, sixth funicle segment.

Charitopus ismailkaracai sp. $\mathbf{n}$.

At least $F_{6}$ less than $2 \times$ as long as broad

Marginal vein shorter than stigmal and at least

$1.5 \times$ as long as postmarginal

Exserted part of ovipositor almost $1.11 \times$ as long

as gaster

Fore and mid femora brown

Stigmal vein $1.4 \times$ as long as postmarginal

leg. GJ \& HC.

Anagyrus schmuttereri Ferriere, 1955

Material examined: PT, 21.V.2009, 1q, leg. GJ \& HC.

**Aphycus moravicus (Hoffer, 1952)

Material examined: AR, 03.IX.2009, 1 , , leg. GJ \& HC.

Blastothrix gurselae Japoshvili and Karaca, 2004

Material examined: AR, 05.X.2009, 1 \%, leg.

GJ \& HC.

Cerapterocerus mirabilis Westwood, 1833

Material examined: AR, 19.VII.2010, 1우, $1 \hat{\jmath}$, leg. GJ.

Charitopus fulviventris Förster, 1860

Material examined: Rocky place, 06.VI.2009, 1§, leg. GJ \& HC; AR, 21.VII.2009, 2 , $2 \hat{\jmath}$, leg. GJ \& HC; AR, 14.VIII.2009, 2 , leg. GJ \& HC; AR, 29.VII.2010, 1 \% , $1 \overbrace{}^{\Uparrow}$, leg. GJ.

Cheiloneurus elegans (Dalman, 1820)

Material examined: PT, 28.V.2009, 1q, leg. GJ \& HC; AR, 14.VIII.2009, 1 q, leg. GJ \& HC; 03,14,24.IX.2009, 3 ㅇ, leg. GJ \& HC.

Cheiloneurus claviger Thomson, 1876

Material examined: AR, 03,12.VII.2009, 2 ㅇ, leg. GJ \& HC; AR, 19.VII.2010, 19, leg. GJ.

Cheiloneurus paralia (Walker, 1837)

Material examined: AR, 14.VIII.2009, 2q, leg. GJ \& HC.

Dusmetia ceballosi Mercet, 1921

Material examined: AR, 03.VII.2009, 1 , leg. GJ \& HC; PT, 03.VII.2009, 1 \%, leg. GJ \& HC; AR, 12.VII.2009, 3 + , leg. GJ \& HC; AR, 21.VII.2009, 2ᄋ, 1今̄, leg. HC; AR, 05.X.2009,
Ch. desertus

All flagellar segments $2 \times$ as long as broad

Marginal vein shorter than stigmal and less

than $1.5 \times$ as long as postmarginal

Exserted part of ovipositor $1 / 6-1 / 7$ as long

as gaster

Fore and mid femora yellow

Stigmal vein more than $2 \times$ as long as postmarginal

1 , , leg. GJ \& HC.

Ericydnus apterogenes Mayr, 1876

Material examined: AR, 21.VII.2009, 1운, leg. HC.

Ericydnus robustior Mercet, 1921

Material examined: AR, 25.VI.2009, 12, $6 \AA$, leg. GJ \& HC; PT, 28.V.2009, 2 , , 1 §, leg. GJ \& HC; AR, 06.VI.2009, 2へ, leg. GJ \& HC; Rocky place, 06.VI.2009, 1 ते, leg. GJ \& HC; AR, 03.VII.2009, 1ठิ, leg. GJ \& HC; AR, 12.VII.2009, 1§, leg. GJ \& HC; AR,

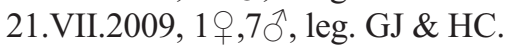

Ericydnus sipylus (Walker, 1837)

Material examined: AR, 25.VI.2009, 3 , 1 1 , leg. GJ \& HC; AR, 03.VII.2009, 5 क, leg. GJ \& HC; AR, 12.VII.2009, 1 q, leg. GJ \& HC; AR, 21.VII.2009, 5q, leg. GJ \& HC; PT, 14.VIII.2009, 1今̄, leg. GJ \& HC; AR, 24.VIII.2009, 1 ㅇ, leg. GJ \& HC; PT, 14.IX.2009, 1 ㅇ, leg. GJ \& HC; AR, 24.IX.2009, 1§, leg. GJ \& HC.

**Leptomastix ephyra Noyes and Hayat, 1994

Material examined: AR, 28.VI.2009, 1 ${ }^{\Uparrow}$, leg. GJ \& HC; AR, 03.VII.2009, 19, leg. HC; AR, 21.VII.2009, 1ð, leg. GJ \& HC; AR, 14.VIII.2009, 19, leg. GJ \& HC; AR, 24.VIII.2009, $2 \hat{\jmath}$, leg. GJ \& HC.

Leptomastix dactylopii Howard, 1885

Material examined: AR, 21.VI.2009, 1ठ, leg. GJ \& HC.

Mahencyrtus comara (Walker, 1837)

Material examined: PT, 28.V.2009, 1ㅇ, leg. GJ \& HC.

**Mayrencyrtus imandes (Walker, 1837)

Material examined: PT, 03.VII.2009, 1 q, leg. 
GJ \& HC; AR, 24.VIII.2009, 19, leg. GJ \& HC; PT, 14.IX.2009, 1 \%, leg. GJ \& HC.

**Metaphycus discolor Hoffer, 1976

Material examined: AR, 21.VII.2009, 1, leg. GJ \& HC; AR, 19, 29.VII.2010, 2ᄋ, leg. GJ.

Mayridia procera (Mercet, 1921)

Material examined: AR, 24.IX.2009, 1ㅇ, leg. GJ \& HC; AR, 29.VII.2010, 19, leg. GJ.

Metaphycus lounsburyi (Howard, 1898)

Material examined: AR, 15.X.2009, 1 9 , leg. GJ \& HC.

Metaphycus petitus (Walker, 1851)

Material examined: AR, 21.VII.2009, 1옹 leg. GJ \& HC.

**Metaphycus stanleyi Compere, 1940

Material examined: AR, 21.VII.2009, 1 + (slide), 1 ( (card mounted), leg. GJ \& HC.

Metaphycus swirskii Annecke and Minhardt, 1979

Material examined: Rocky place, 06.VI. 2009, 2 , leg. GJ \& HC.

Metaphycus zebratus (Mercet, 1917)

Material examined: AR, 12.VII.2009, 2q, leg. GJ \& HC.

**Microterys darevskii Trjapitzin, 1968

Material examined: AR, 19.VII.2010, 1옹 leg. GJ.

Pseudococcobius obenbergeri (Novickij, 1926)

Material examined: AR, 21.VII.2009, 1\%, leg. GJ \& HC; 14.VIII.2009, 2q, leg. HC; 19.VII.2010, 1 ㅇ, leg. GJ.

Rhopus flavidus (Mercet, 1921)

Material examined: PT, 28.V.2009, 1q, leg. GJ \& HC; AR, 12.VII.2009, 1ㅇ, leg. GJ \& HC.

Zaomma lambinus (Walker, 1838)

Material examined: AR, 03.IX.2009, 1 ㅇ, leg. GJ \& HC.

Acknowledgments. I would like to thank to Prof. Dr Roy Van Driesche and Dr. Kristopher Abell, Department of Plant, Soil, and Insect Science, University of Massachusetts, MA, USA, who kindly helped check the English and gave advice on improvements for this paper. Thanks to David Kvitaishvili and Giorgi Giorganashvili for their help in preparing figures.

\section{References}

Guerrieri, E. \& Noyes, J. S. 2000: Revision of European species of genus Metaphycus Mercet (Hymenoptera: Chalcidoidea: Encyrtidae), parasitoids of scale insects (Homoptera: Coccoidea). — Systematic Entomology 25: $147-222$.

Hayat, M. 2006: Indian Encyrtidae (Hymenoptera: Chalcidoidea). — Department of Zoology, Aligarh Muslim University, Aligarh. 496 pp.

Japoshvili, G. O. 2005: Anew species of encyrtid, Psilophrys ghilarovi (Hymenotpera, Chalcidoidea, Encyrtidae) from Turkey. - Zoologicheskyi Zhurnal 84: 524-527. [In Russian.]

Japoshvili, G. 2007: New data on species of Syrphophagus (Hymenoptera: Encyrtidae) from Transcaucasus and Turkey. - Annals of the Entomological Society of America 100: 683-687.

Japoshvili, G. \& Karaca, I. 2002: Coccoid (Homoptera: Coccoidea) species of Isparta province and their parasitoids from Turkey and Georgia. — Turkish Journal of Zoology 26: 371-376.

Japoshvili, G. \& Karaca, I. 2004 : New record of encyrtid parasitoids of Kermes palestiniensis Balachowsky (Hemiptera: Kermesidae), with the description of a new species of Blastothrix Mayr (Hymenotpera: Encyrtidae) from Turkey. — Entomological news 114: 187-191.

Japoshvili, G., Karaca, I., Ulgenturk, S., Demirozer, O. \& Kaydan, B. 2004: Encyrtids (Hymenoptera, Encyrtidae) parasitoids of Coccoids (Homoptera, Coccoidea) from Turkey. - Proceedings of the Institute of Zoology, Georgian Academy of Sciences 22: 188-192. [In Russian.]

Japoshvili, G. \& Noyes, J. 2005a: Checklist and new data on Encyrtidae of Transcaucasia and Turkey (Hymenoptera: Chalcidoidea). — Zoosystematica Rossica 14: 135-144.

Japoshvili, G. \& Noyes, J. 2005b: New record of Encyrtids (Hymenoptera: Chalcidoidea). — Kavkazskiy Entomologicheskiy Bulleten 1: 159-160.

Japoshvili, G. \& Abrantes, I. 2006: New records of Encyrtids (Hymenoptera: Chalcidoidea; Encyrtidae) from Portugal, Including Descriptions of Two New Species. - Entomological News 117: 423-431.

Japoshvili, G. \& Noyes, J. 2006: The Western Palaearctic species of Psilophrys Mayr (Hymenoptera, Chalcidoidea: Encyrtidae), parasitoids of kermesids (Hemiptera, Coccoidea: Kermesidae) attacking oaks (Quercus spp.). - Journal of Natural History 40: 1783-1800.

Japoshvili, G. \& Celik, H. 2010: Fauna of Encyrtidae (Hymenoptera: Chalcidoidea), parasitoids of Coccoids (Hemiptera: Coccoidea) in Golcuk Natural Park. Entomologia Helenica 19: 132-136.

Karaca, I. \& Japoshvili, G. 2002 : An annotated list of the chalcid (Hymenoptera: Chalcidoidea) parasitoids of Coccoids (Hemiptera: Coccoidea) in Isparta Province (Turkey). - Proceedings of the Institute of Zoology, Georgian Academy of Sciences 21: 173-175. 
Kaydan, M. B., Kilincer, N., Uygun, N., Japoshvilli, G. \& Gaimari, S. 2006: Parasitoids and Predators of PseudoCoccoidae (Hemiptera: Coccoidea) in Ankara, Turkey. - Phytoparasitica, 2006, 34: 331-337.

Kaydan, B. \& Japoshvili, G. 2010: The aphelinid and encyrtid parasitoids (Hymenoptera: Chalcidoidea) of PseudoCoccoidae (Hemiptera: Coccoidea) in the Van Lake basin of Turkey. - Turkish Journal of Entomology 34: 465-476.

Noyes, J. 2010: Universal Chalcidoidea Database. [www document] URL www.nhm.ac.uk/entomology/chalcidoids/index.html (Site visited on 2 September, 2010).

Noyes, J. \& Hayat, M. 1994: Oriental mealybug parasitoids of the Anagyrini (Hymenoptera: Encyrtidae). CAB International, Oxon, UK. 554 pp.

Öncüer, C. 1991: A catalogue of the parasites and predators of Turkey. - Publication of Ege University, Faculty of Agriculture No: 505, Izmir. 354 pp. [In Turkish.]

Townes, H. 1972: A light-weight Malaise trap. — Entomological news 83: 239-247.
Trjapitzin, V. A. 1989: Parasitic Hymenoptera of the Fam. Encyrtidae of Palaearctics. — Opredeliteli po Faune SSSR. Zoologicheskim Institutom Akademii Nauk SSR, Leningrad. 488 pp.

Trjapitzin, V. \& Doganlar, M. 1997: A review of encyrtids (Hymenoptera, Encyrtidae) of Turkey. - Entomologicheskoe Obozrenie 76: 213-222.

Uygun, N., Oncuer, C., Karaca, I., Erkilic, L., Yoldas, Z., Sengonca, C. \& Japoshvili G. 2004: An Annotated List of the Natural Enemies of the Scale Insect (Homoptera: Coccoidea) of Turkey. - Proceedings of the Institute of Zoology, Georgian Academy of Sciences 22: 173-185.

Yasnosh, V. \& Japoshvili, G. 1999: Parasitoids of the genus Psyllaephagus Ashmead (Hymenoptera: Chalcidoidea: Encyrtidae) in Georgia with the description of $P$. georgicus sp.n. - Bulletin of the Georgian Academy of Sciences 159: 516-519. 